UCRL-ID- 118354

\title{
The Future of Finite Element Applications on Massively Parallel Supercomputers
}

\author{
Mark A. Christon \\ Methods Development Group \\ Lawrence Livermore National Laboratory \\ P.O. Box 808 \\ Livermore, California 94550
}

July 5, 1994

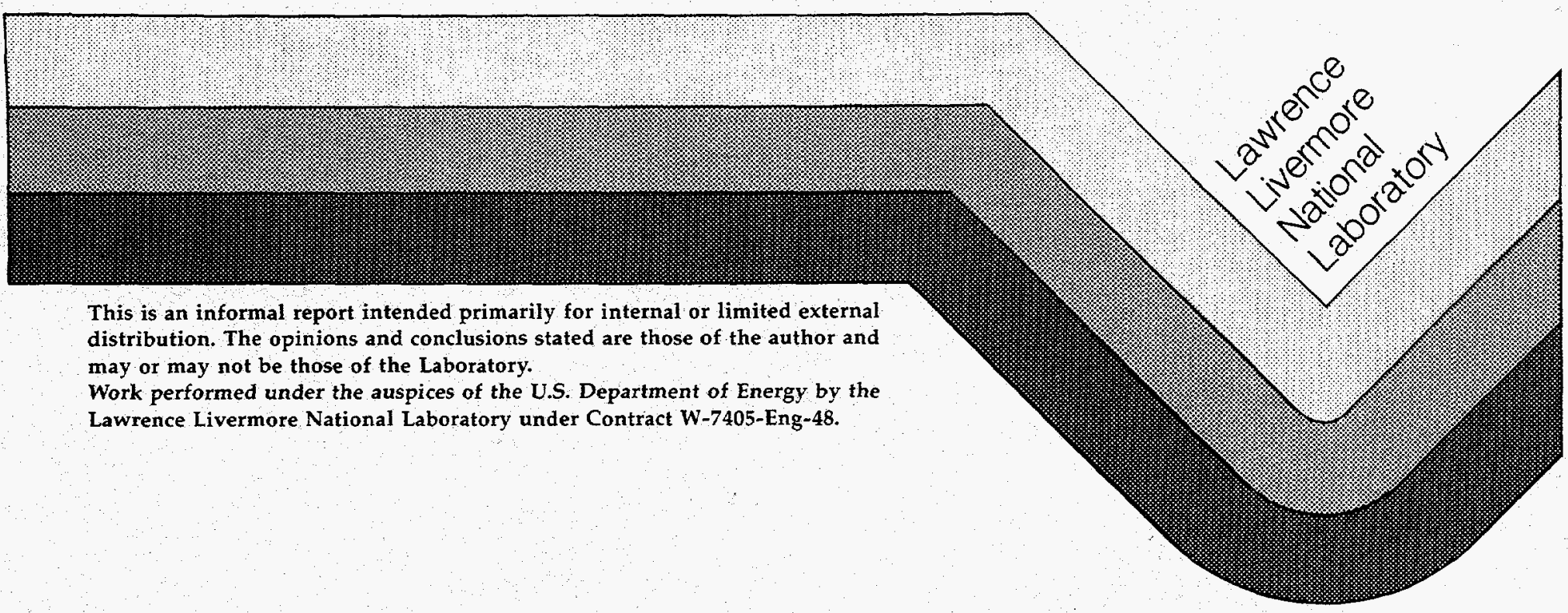




\section{DISCLAIMER}

This document was prepared as an account of work sponsored by an agency of the United States Government. Neither the United States Government nor the University of California nor any of their employees, makes any warranty, express or implied, or assumes any legal liability or responsibility for the accuracy, completeness, or usefulness of any information, apparatus, product, or process disclosed, or represents that its use would not infringe privately owned rights. Reference herein to any specific commercial products, process, or service by trade name, trademark, manufacturer, or otherwise, does not necessarily constitute or imply its endorsement, recommendation, or favoring by the United States Government or the University of Calfornia. The views and opinions of authors expressed herein do not necessarily state or reflect those of the United States Government or the University of California, and shall not be used for advertising or product endorsement purposes.

This report has been reproduced

directly from the best available copy.

Available to DOE and DOE contractors from the

Office of Scientific and Technical Information

P.O. Box 62, Oak Ridge, TN 37831

Prices available from (615) 576-8401, FTS 626-8401

A vailable to the public from the

National Technical Information Service

U.S. Department of Commerce

5285 Port Royal Rd.

Springfield, VA 22161 


\title{
The Future of Finite Element Applications on
}

\section{Massively Parallel Supercomputers}

\author{
Mark Christon \\ Methods Development Group, L-122 \\ Lawrence Livermore National Laboratory \\ P.O. Box 808 \\ Livermore, California 94550
}

July 5, 1994

Abbreviated Title : MPC FEM Applications

DISTRIBUTION OF THIS DOCUMENT IS UNLIMITED

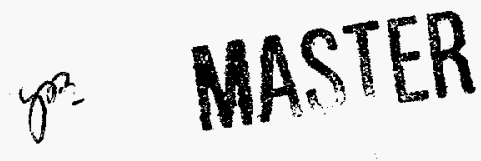




\begin{abstract}
The current focus in large scale scientific computing is upon parallel supercomputers. While still relatively unproven, these machines are being slated for production-oriented, general purpose supercomputing applications. The promise, of course, is to use massively parallel computers to venture further into scientific realms by performing computations with anywhere from $10^{6}$ to $10^{9}$ grid points thereby, in principle, obtaining a deeper understanding of physical processes. In approaching this brave new world of computing with finite element applications, many technical issues become apparent. This paper attempts to reveal some of the applications-oriented issues which are facing code developers and ultimately the users of engineering and scientific applications on parallel supercomputers, but which seem to be remaining unanswered by vendors, researchers and centralized computing facilities. At risk is the fundamental way in which analysis is performed in a production sense, and the insight into physical problems which results. While at first this treatise may seem to advocate traditional register-to-register vector supercomputers, the goal of this paper is simply an attempt to point out what is missing from the massively parallel computing picture not only for production finite element applications, but also for grand challenge problems. The limiting issues for the use of FEM applications on parallel supercomputers are centered about the need for adequate disk space, archival storage, high bandwidth networks, and continued software development for mesh generation, scientific visualization, linear equation solvers and parallel input/output.
\end{abstract}

\title{
1 Introduction
}

The term supercomputer has traditionally been reserved for the largest, fastest, and also the most expensive computers in the world. These machines have been the largest in terms of memory, the fastest in terms of delivered, 64-bit, floating point operations, and the most expensive not only in terms of their initial purchase price but also in their operating cost. On the other hand, the term "massively parallel" computer means different things to different people. To the community of seasoned supercomputer users, the term massively parallel computer (MPC) is understood to mean machines with greater than $1024(1 \mathrm{~K})$ processors. 


\section{DISCLAIMER}

Portions of this document may be illegible in electronic image products. Images are produced from the best available original document. 
In terms of memory and promised peak performance, currently available parallel computers such as the Thinking Machines CM-5, the Meiko CS2 , and the INTEL Paragon meet the criteria of being supercomputers not only in terms of promised peak performance, but also in terms of memory and price. However, few installed parallel systems really meet the criteria of being massively parallel (i.e., greater than $1 \mathrm{~K}$ processors), so the $M$ in MPC should really be taken to mean moderately - both in this paper, and in practice. This is so because most delivered systems, with the notable exception of the Los Alamos CM-5, have fewer than $1 \mathrm{~K}$ processors and still meet the definition of a supercomputer.

In terms of peak performance, those who are experienced with supercomputer applications agree that peak performance refers to the level of floating point performance which the vendor guarantees the end user will never be able to obtain. Unfortunately, the disparity between the delivered floating point operation (FLOP) rate, and the vendor's promised peak FLOP rate varies drastically across supercomputer architectures and applications. The ability of a supercomputer to deliver a large fraction (say $50-60 \%$ ) of its peak performance on a wide variety of applications is a measure of its capability as a general purpose computing resource.

Over the past 15 years, FEM codes have been developed which exploit the register-to-register vector architectures which have popularized supercomputing among the masses. The fact that CRAY provided specialized hardware for gather/scatter operations has made it possible for the unstructured grid performance of FEM codes to be very good since all FEM codes require gather/scatter operations for the assembly of element-to-global data at some point (independent of the code). Highly vectorized FEM codes are represented by the LLNL codes DYNA3D [1] and NIKE3D [2]. In general, a vectorized FEM code can easily achieve approximately 25 to $50 \%$ of the peak single processor performance of a CRAY Y-MP (the range of 85 to 170 MFLOPs) with only a moderate effort on the developer's part (e.g., [3]). However, it is estimated that computational resources providing from $\sim 10$ GigaFLOPs up to $\sim 1$ TeraFLOP ( 1 trillion floating point operations per second) will ultimately be necessary to perform simulations for viscous, turbulent flow where complex three-dimensional geometry is involved. 


\section{$1.1 \quad$ FEM Applications}

FEM codes for time dependent problems are usually categorized as either implicit or explicit (e.g., NIKE3D is implicit, DYNA3D is explicit). Explicit finite element codes usually exploit some form of mass lumping (diagonalization of the FEM mass matrix) and therefore do not require the solution of any linear systems. Explicit codes only require gather/scatter operations for the assembly of the right-hand-side nodal terms from element level contributions. Explicit engineering mechanics FEM codes have small memory requirements and are highly vectorizable, but do not always achieve the peak performance on vector supercomputers because of the poor ratio of operations to loads/stores. Note that the best performance of register-to-register vector supercomputers is achieved when the ratio of operations to loads/stores is maximized with respect to the size and number of vector registers and the full memory bandwidth of the machine is used. Most explicit codes have a low ratio of operations per load/store, and do not make use of the memory bandwidth. However, the performance of explicit FEM algorithms can be improved if sufficient physics (i.e., computational work) is available.

In contrast, implicit FEM codes generate large sparse, banded, and usually symmetric linear systems which must be solved at each time step. Many iterative and direct linear system solvers are available for register-to-register vector supercomputers and are able to achieve very high floating point performance on CRAY architectures [4]. For this class of FEM code, the hardware gather/scatter is required not only for the assembly of the right-hand-side terms, but also in the formation of the sparse linear operator on the lefthand-side. The implicit class of FEM codes can achieve very high FLOP rates, in part due to the readily available high performance linear solvers which maximize the ratio of operations to loads/stores and truly use the full memory bandwidth of the CRAY architecture. However, the cpu time per element per time step is usually much lower than can be achieved with an explicit code. Vectorization techniques for FEM codes are well known, and have permitted the application of general purpose codes to broad classes of problems on CRAY architectures for over 15 years. DYNA3D/NIKE3D are representative of the type of finite element applications which have given rise to the idea of production supercomputer codes. 


\subsection{FEM Analysis and Production Computing}

The idea of production supercomputer applications involves the notion that an analyst who is not a code developer, algorithm researcher, or applied mathematician, can make use of supercomputer applications for mesh generation, simulation and visualization in an efficient way to solve scientific and engineering problems. CRAY-based production computing environments have led to a methodology for ananysis which is at risk in light of the current class of parallel supercomputers. In this model, the analysis cycle consists of the generation of a finite element mesh complete with boundary conditions, initial conditions, and material definitions. Additionally, a description of the number of time steps to be taken and the intervals to output time history data, complete state data (a snapshot of the simulation at the current state in time), restart dumps, etc., are produced during the mesh generation phase. The simulation is performed and the state data and time history data are visualized, synthesized, and a decision made upon mesh adequacy, modified analysis parameters, and then another simulation is performed. Typically, this process involves on the order of 5-10 simulation runs with varying mesh resolution or analysis parameters to ensure solution accuracy.

The process of performing simulations in this way can be viewed as a form of computational experimentation where the engineer or scientist is probing the sensitivity of a physical process to specific parameters. In this process, the state data, which contains the primary solution variables (e.g., velocity, vorticity, pressure) at a given point in time, are written to temporary disk along with time history data at a small number of grid points. Usually the time history data are recorded every time step, while a coarser sampling interval is used for the state data. The methodology of archiving these datasets for later interrogation has become standard not only at the DOE national laboratories (LANL, LLNL, SNL), but also at the NSF sponsored supercomputing centers such as the National Center for Supercomputing Applications (NCSA), the Pittsburgh Supercomputer center (PSC), and the San Diego Supercomputer Center (SDSC).

The ability to probe and interrogate data is a key part of not only understanding the results of a simulation, but also in verifying its correctness. Visual debugging is the use of advanced visualization tools which can aid the user in not only understanding data, but also in identifying computational defects such as improperly specified boundary conditions. Because a general 
purpose supercomputer code can be applied to a spectrum of problems, it is very difficult to decide upon specific output data a priori for the sake of reducing state dataset size. Indeed, often it is the case that during the interrogation of simulation results, the visualization study may motivate the user to look at many different derived variables, e.g., effective stress, strain rate, etc. Data abstraction in a sophisticated visualization tool assumes that sufficient primitive data is available to compute the necessary results, i.e., the state data can't be gutted for the sake of disk space. It may require on the order of days to fully explore results from a large scale computational fluid dynamics or computational solid mechanics simulation with a suitable understanding of the results. This requires the ability to rapidly recover a dataset from archival storage.

This paper assesses the architectures of two MPC's relative to traditional vector supercomputers, how they relate to FEM codes, and the performance of FEM codes on these machines. The requirements for mesh generation, linear equation solving, visualization, storage and networks is analyzed with respect to the application of finite element tools to grand challenge problems [5].

\section{MPC Architectures}

The truth about the architecture and performance characteristics of MPC's is sometimes difficult to obtain. The economic competitive demands on supercomputer vendors are extreme, and the newness and novelty of many parallel computers makes it possible for misunderstandings to occur [6]. The variety of computer architectures and the jargon used to describe these machines (SIMD: Single Instruction Multiple Data, MIMD: Multiple Instruction Multiple Data, shared memory, distributed memory, omega network, fat tree, etc.) make it difficult to evaluate computer performance in a simple way. Further, the interpretation of performance data in terms of applications is confused by the multiple programming models which are available today, e.g., domain decomposition message passing (DDMP) vs. data parallel (F90).

A good example of this type of misunderstanding is demonstrated by the vector performance of the Thinking Machines CM-5. In the case of the CM5 , vendor literature states that there are 4 vector pipelines per processor, each pipeline effectively having a vector length of 8 . However, attempts to 
use effective vector lengths of 8 results in very poor performance, while using vector lengths of 1024 results in reasonable performance, although still far from the reported peak FLOP rate. While the CM-5 architecture seems to be quite new, the machine is little more than an array processor whose vector half-length is proportional to the number of processors [7]. The vector halflength is the vector length required to achieve $50 \%$ of the asymptotic FLOP rate of the vector processor. To be specific, measured data show that the vector half-length for the CM-5 without the vector pipelines is essentially that for an array processor:

$$
N_{1 / 2}=\frac{N_{p}}{2}
$$

where $N_{1 / 2}$ is the vector half-length, and $N_{p}$ is the number of processors. For the CM-5 with vector units; the vector half-length is approximately:

$$
N_{1 / 2} \approx 4 N_{v \psi} N_{p}
$$

where $N_{v u}$ is the number of vector units per processor. Fig. 1 shows the timing results used for estimation of the CM- 5 half-lengths.

Further, data motion (i.e., gather/scatter operations) degrades the FLOP rate by a factor of 3 to 10 times depending on the specific code implementation. (This performance degradation was measured for indexed,,$+- *$ operations relative to the same operations performed with a unit stride.) Indeed, the most successful applications on the CM-5 are finite difference codes whose algorithms can be cast into a succession of very long vector operations with unit strides (very long with respect to register-to-register vector supercomputers). This is not too surprising since $N_{1 / 2}$ is essentially a measure of the parallelism in a given computer architecture. In contrast, $N_{1 / 2}$ may also be interpreted as an indication of how specialized the computer is. That is, a general purpose computer will have a relatively low $N_{1 / 2}$. In the case of the CM-5, $N_{1 / 2}$ reflects the fine-grained nature of the architecture. Similar findings have been reported from the ParaDyn project [9].

By comparison, the Meiko CS-2 documentation for the Fujitsu vector units states that the vector registers may be reconfigured to accommodate vector lengths from 8 to 256 [8]. This suggests that the vector pipeline can accommodate a wide variety of vectorizable loops - loops ranging from short and simple to long and relatively complex. However, the fact that $\mathrm{O}(100)$ 
clock cycles are required to initiate vector instructions means that the effective vector start-up time is about 2 times worse than the CDC Cyber 205/ETA-10. Unfortunately, one of the more serious defects of the Cyber 205/ETA-10 was the fact that a vector add or multiply required about 51 clock cycles to start-up. The long start-up times on the Cyber 205, the requirement for long vectors $\left(N_{1 / 2} \approx 100\right.$ for a two-pipe machine), the performance degradation for non-unit strides, and generally poor scalar performance made it relatively undesirable for most FEM codes. This is especially true when compared to the CRAY architecture with it's short vector start-up and $N_{1 / 2} \approx 10$.

Given the time to initiate vector operations on the CS-2, a two-pipe processor of the Meiko can be expected to have $N_{1 / 2} \approx 100$. The relatively long effective vector start-up on the CS-2 implies that long vectors will be necessary to achieve a reasonable fraction of the 200 peak MFLOPs on a single processor (assuming that the well known cache coherency problem has been rectified). That is, relatively long vectors will be required to amortize the cost of initiating a vector operation. However, the peak theoretical scalar-tovector speedup is only 5 [18], i.e., a code must be $100 \%$ vectorized to achieve a speedup of 5 . While machines such as the CS-2 may not offer optimal vector characteristics for FEM codes, they do offer promise because of their good scalar performance (cached RISC architecture) and good network bandwidth. The network bandwidth of the Meiko CS-2 is approximately 5 times greater than the CM-5 network bandwidth which is important in light of the concomitant communication required for unstructured grid applications.

It should be noted that codes such as DYNA3D, NIKE3D, PING [10] and HYDRA [11] use the concept of vector blocking for compute intensive operations making it a simple task to configure the vector block size according to the cache size of the SPARC based processors of the CS-2. This approach results in optimal code for this class of RISC processor. While the current Methods Development Group (MDG) codes may easily be configured for specific computer architecture attributes such as cache size, there are studies which suggest that code tuning for machines such as the CM- 5 actually result in better performance on CRAY machines when the tuned applications are moved back to the CRAY [12] - a situation which only complicates the problems of adequately assessing the performance of FEM codes on new architectures.

By comparison, MIMD machines are proving quite adequate for discrete 
event simulations which are very difficult to vectorize but are embarrassingly parallel, i.e., they can be easily parallelized and require little communication. Discrete event applications have always been completely scalar and only vectorized with valiant efforts [13]. In fact, in many circumstances, scalar processors such as the IBM RS-6000 have come very close to delivering the performance of CRAY's on these type of applications. Unlike discrete event codes, the nature of unstructured grid applications is that they require off-processor communication for the same reason that they require gather/scatter operations on register-to-register vector supercomputers. Therefore, the Meiko CS-2 provides the best network bandwidth of the available MPC's in its class which makes the CS-2 a good choice for FEM applications in spite of its shortcomings for single processor vector processing. The promise of the Meiko CS-2 is reflected in the early results of the ParaDyn project with DYNA3D [9].

\section{Mesh Generation}

Implicit in the FEM community is the fact that the term "mesh" is usually taken to mean the set of discrete nodes and elements for the physical domain and all the boundary conditions, initial conditions, material properties and analysis description necessary to perform a simulation. Unlike academic finite difference applications, which consider a set of grid points arranged in a block logical fashion to be sufficient for an analysis, FEM applications require additional information to describe the elements, how nodes are connected to elements, boundary conditions, etc.

There are many types of mesh generation techniques available today. However, the primary methodology in use in a production sense is the blockstructured method which relies upon either algebraic or elliptic fill algorithms, e.g., codes like TrueGrid, Eagle, etc. While plastering, paving, elliptic and hyperbolic methods have been available for quite some time, the majority of mesh generators rely heavily upon the computationally efficient algebraic multi-block methods. Typically, relatively complicated meshes, both in terms of geometry and number of parts, may be constructed efficiently with algebraic methods on the current generation of workstations. However, multi-block algebraic methods do not permit the user a great deal of flexibility in generating 3-D transitions from coarse to fine mesh regions, and do 
not exploit the true unstructured nature of the analysis codes. However, an adequate job may be done on moderate size meshes with the current tools.

The compute requirements of an algebraic multi-block mesh generator doesn't demand MPC FLOP rates, so why is parallel mesh generation important? The current answer is because a workstation can't support disk space sufficient to hold a mesh for a large parallel simulation $\left(10^{8}\right.$ grid points would require about $5-10$ GBytes of disk, $10^{9}$ grid points about $50-100$ GBytes) Because the MPC meshes will be too large for current workstation disks, the current thinking is to compute the mesh in blocks on the MPC machine and store a distributed version of the mesh on processor-local disks. The idea of writing the mesh block on multiple processor-local disks exploits one of the key advantages of the Meiko CS-2. However, the current processorlocal disk space is limited to approximately of 1/2 GByte. Thus on a 256 processor machine, a mesh requiring 100 GBytes of disk space could easily swamp the available disk storage leaving no available space for the simulation results. Clearly, re-assembling a sequential mesh file on the global file system is a waste of computer resources since the sequential file would still require the same storage, and it would ultimately have to be re-distributed for the simulation.

The trend for mesh partitioning for parallel simulation is toward spectral methods such as recursive spectral bisection (RSB) $[14,15,16]$ to intelligently and automatically sub-divide an existing mesh and map the parts onto a parallel machine in such a way that communications overhead will be of second-order importance in the cost of the computations. Currently, if the mesh is large, a CRAY is required to deliver the necessary punch required to recursively compute the second eigenvalue of the graph of a mesh with $\mathrm{O}\left(10^{6}\right)$ nodes. This leaves the question, are spectral partitioning methods going to provide an adequate means for partitioning when the process is limited by the ability to compute the eigenvalues of the mesh graph on a CRAY? Parallel partitioning schemes are not available yet, in part, due to the starting point for such a partitioning algorithm which is a sequential data file containing the graph of a mesh. Secondly - software for performing eigenvalue analysis on a parallel machine is limited due to the lack of adequate linear equation packages which are fast and can treat the sparse nature of the linear systems generated by unstructured grid methods (Note the nearly complete omission of sparse solvers in the CM-5 Mathematical Library CMSSL [17]).

Ideally the mesh partitioning should be a part of mesh generation, but 
the optimal distribution for block structured mesh generation may not always provide an optimal distribution for the analysis. For example, for load balancing considerations, a parallel multi-block algebraic mesh generator would attempt to distribute blocks with an equal number of nodes and elements on each processor. At first glance, this seems reasonable for the analysis as well. However, for computational mechanics, where a mesh may consist of beams, shells, and brick elements each of which has a different computational load, such a distribution of elements is not adequate for the analysis code. In the case of flow past a submarine, the geometrical complexity of the submarine hull, and the mesh resolution requirements for the boundary layer may require a very different geometrical decomposition for the mesh generation process than would be optimal for minimizing communication in the simulation. The idea of re-partitioning and re-distributing the mesh after it has been generated on a large number of processors is problematic and an area of ongoing research (e.g., the LDRD funded Parallel Mesh Generation effort [19]).

Another issue to be attacked in the context of mesh generation for grand challenge problems is that of mesh visualization. Before launching a computation on a mesh with $10^{6}$ to $10^{9}$ grid points a great deal of confidence in the problem definition is required. The ability to interactively interrogate element and nodal data in the mesh, as well as, boundary conditions, initial conditions, sliding interfaces, material boundaries, etc. is key to the effective generation of FEM meshes. However, the idea of generating a multi-block mesh with each block on a different processor of an MPC poses many problems for effective visualization of the mesh. Clearly, the mesh visualization process must be interactive and integrated with the mesh generation process. In light of all this, how will an analyst make use of an MPC mesh generator in an interactive way? This issue will be revisited in the discussion on scientific visualization below.

\section{Linear Equation Solvers}

Many finite element applications require the solution of one or more linear systems at each time step in a simulation. The state of linear equations solvers. on distributed memory parallel computers is lagging because of the difficult communications issues which arise in a direct solution algorithm 
such as Gaussian elimination. (In contrast, a good deal of success has been achieved with linear systems solvers on shared memory parallel supercomputers such as the CRAY Y-MP [20].) Further, it is widely held that the roundoff error with direct methods on linear systems with $10^{6}$ to $10^{9}$ equations will make these methods unusable even with 64-bit arithmetic. All of this has pushed the linear equation solving towards iterative methods because they are easier to parallelize without incurring high communications overhead. Further, the availability of robust direct equation solvers for sparse, banded, distributed systems is lacking - with the notable exception of Scalapack [22]. The Methods Development Group is currently pursuing a small research effort with the PVS solver [4] on the Meiko CS-2, but the work is still in a preliminary stage.

While there has been much research on solving $\Delta \phi=0$ on a unit square with iterative techniques, the demands for robust preconditioners for iterative solvers in unstructured grid FEM applications are going seemingly without notice. The demands of structural codes are quite different from CFD codes even though the data structures can be identical for both. For example, a turbulent flow simulation may require the use of boundary layer elements with aspect ratios as high as $10^{5}$, while a structural simulation may require the use of a heterogenous mesh comprised of beam, shell and brick elements. Ultimately, this means that a preconditioner which is adequate for $\Delta \phi=0$ on a unit square will probably not be suitable for an FEM application, and a preconditioner for a structural application may not be well suited for a fluid dynamics problem. As with direct solvers, preconditioners which use a form of incomplete factorization are ultimately limited by poor parallel efficiency due to communications overhead [21]. The bottom line is that explicit FEM applications will undoubtedly be the first applications on MPC machines, while implicit FEM applications will be delayed or forced to run with poor performing preconditioners and iterative solvers until adequate equation solvers (preconditioners) are available.

\section{Scientific Visualization}

The goal of scientific visualization is to enable the rapid assimilation of large amounts of data produced by scientific and engineering simulations. Effective visualization hinges on the ability to interactively probe and interrogate data 
to the fullest extent. Scientific visualization requirements for grand challenge problems ( $10^{6}$ to $10^{9}$ grid points) pose many difficulties in light of the current generation of parallel computers. In the model of analysis whereby the process of mesh generation, simulation, and visualization may be repeated several times as part of a computational investigation, the implicit assumption is that there will be sufficient disk space available to write the required data for a subsequent visualization study.

In a visualization study, derived quantities such as stress components, effective stress, vorticity, wall shear stress, etc. are required for data abstraction. It is important to note that the investigator may not know a priori that visualization of a specific quantity, e.g., effective stress, is even required until after some initial interrogation of the data has been performed. This requires two things: first, the original primitive data must be available for the computation of the derived variables, and second, there must be sufficient compute power to perform the necessary operations for the visualization process. While this is usually the case for traditional CRAY supercomputers (and the problem size which they can handle), when considering the increased number of grid points which will be used on MPC's, the available disk space on these machines is far from adequate.

Consider the example of an acoustic fluid-structure interaction simulation where a highly discretized fluid region is required. PING [10] output is usually in the form of 3 pressure components: the incident pressure, the elastic-scattered pressure, and the total perturbation pressure (neglecting the smaller number of structural displacements, velocities and accelerations). Assuming for the moment that 32-bit floating point values are sufficient, and neglecting the data defining how nodes are connected to elements, the number of states to faithfully represent temporal wave-like phenomenon in the pressure field as set by the Nyquist theorem can be estimated in terms of the grid frequency.

The term "grid frequency" refers to the highest discrete frequency which can be supported by a given mesh. The Nyquist theorem states that a sampling frequency twice the highest frequency of interest must be used to avoid temporal aliasing. This translates into the number of states of data which must be saved if an accurate animation of wave propagation is to be created. For the 256 processor Meiko CS-2, upwards of $10^{6}$ node simulations will be tractable in terms of the necessary FLOP rate and available memory per processor. However, the disk requirements for such a problem (shown in 
Table 1) may make the data management difficult and possibly intractable whan scaling up to problems with $10^{9}$ grid points. Clearly, if visualization is to occur after the simulation, and permit the computation of derived results, then from 0.8 to 80 GBytes of disk space will be required. Thus, the total amount of disk storage required for a simulation will be the sum of the storage for the mesh, the state datasets, and the time history data.

Of course, this estimate of required disk space assumes that the temporal behavior is of enough interest to prompt the investigator to look at transient phenomena. This estimate also assumes that the visualization study will make use of the high quality volume data which was computed on the MPC. For example, isosurfaces of the pressure field in the volume of the discrete domain will be visualized, as opposed to essentially gutting the entire volume of data in favor of performing visualization studies on the external boundary of the domain.

The reason for the interest in the interior volume data stems from several things. First, the MPC simulation was performed to obtain all the data, and discarding the interior volume of data seems to be a gross waste of both time and money in terms of the computer resources expended in obtaining the data. Second, visualizing boundary data is not interesting when the boundary conditions are well known and specified. The interesting and unknown results are usually in the volume of data for which the computation was performed. Thus, the paradigm shift of "dataset gutting" does not appeal to the user who is interested in solving real problems. In fact, the idea of gutting datasets clearly falls in the category of ways to say nothing with scientific visualization (see Globus [23]).

Another alternative for visualization is the idea of co-processing as proposed by Haimes [24]. The basic concept is that because it will not be possible to store the 10 - 100 GBytes of data from the simulation, the visualization must be done on the fly while the simulation is running. Of course, this forces the computational part of the visualization software to run on the MPC concurrently with the simulation. One difficulty with this approach is that the user must select the data and derived variable to be visualized at the time the simulation is run. Because the data is essentially viewed and discarded, any change in the derived variable requires the simulation and visualization to be re-run. Clearly for a grand challenge problem which requires all 256 processors of the CS-2, re-running problems just because the user wishes to view velocity magnitude instead of a vorticity component is 
impractical. Such a paradigm shift for analysis and visualization is in vogue, but the model is only suitable for the hero model of supercomputing [25].

While the hero model may be workable under certain circumstances, the use of MPC's for production, general purpose computing will necessitate the intelligent use of resources. The idea of graphical extracts [26] for visualization of transient computational fluid dynamics problems makes use of the ability to communicate primitive data to a high performance graphics workstation for rendering. This approach requires a distributed visualization application where the $\mathrm{I} / \mathrm{O}$ and compute intensive portion of the visualization application runs on the MPC, and only primitive data such as polygons and derived data are communicated to the workstation. In this visualization model, the amount of data to be moved to the workstation is reduced to a workable level, and the method avoids gutting volume data. Further, this mode of visualization permits the user to perform geometric transformations (i.e., rotate, translate, scale), as well as operations such as color palette manipulation interactively on the workstation.

In the distributed, parallel implementation of a visualization tool here at LLNL, it is assumed that the processor-local disk space of the Meiko CS-2 will be used. While this is currently limited to approximately $1 / 2$ GByte per processor, the advantages of the processor-local disk space are signifcant. The processor-local disk is key because the re-assembly of sequential state datasets is a sequential bottleneck in the parallel FEM application. Further, the visualization of a grand challenge problem will require parallel computations and would require re-partitioning and re-distributing the data for visualization if a sequential database were constructed. Further, not even a super-workstation will handle the sequential dataset for a problem with greater than $10^{6}$ grid points. Many graphics operations such as the computation of isosurfaces, cutting planes, and vector plots, are embarrassingly parallel. Thus if each processor writes a stand-alone graphics state dataset on the processor local disk, there is no sequential bottleneck for the analysis code, and the visualization process can easily exploit the distributed state data. For example, using processor local stand-alone state datasets, the user may wish to first interrogate results on an interesting subdomain before attempting to a global view of the data.

Current ethernet speeds are sufficient to support point-to-point communication of approximately 10,000 polygons per second (assuming a polygon consists of a set of 4 vertex coordinates, 4 vertex normals, and 1 scalar derived 
quantity per vertex). The real difficulty is with the network load when many processors are trying to communicate with a single workstation. Interactivity may ultimately dictate the use of Gigabit networks for parallel distributed visualization in a production environment where an MPC is serving a group of users rather than an individual in the hero model.

The difficulty with this model is that there must be sufficient disk space available on the MPC to hold the state data from a transient simulation, and the network bandwidth must be sufficient to permit the communication of a relatively large number of polygons from multiple processors of the supercomputer to the workstation. A transient simulation which fills the physical memory of a supercomputer will produce a quantity of data which is proportional to the number of time steps times the physical memory of the computer. For example, a grand challenge (hero) problem may require all of the memory of the 256 processors of the Meiko CS-2 and take $10^{3}$ to $10^{4}$ time steps. Assuming that only $10 \%$ of the data is actually required to perform the subsequent visualization, animation, etc., a total of 3.27 TeraBytes (TBytes) of disk would be required, and an additional 3.27 TBytes of archival storage would be necessary. Today, this amount of disk capacity is not available on the Meiko. However, the National Storage Laboratory is investigating technologies with TeraByte capacity, and hopefully these technologies will be integrated in the final Meiko CS-2 configuration.

\section{Summary and Conclusions}

The issues regarding the use of parallel supercomputers for both grand challenge problems and routine production computing are somewhat involved and are related in general to the need for additional disk space, high bandwidth networking, and continued software development in the areas of mesh generation, mesh partitioning, scientific visualization, linear equation solvers, and efficient input/output (I/O). The issues which will hamper the progress of FEM applications on MPC's are itemized below.

- The processor-local disk space of the Meiko CS-2 is one of its key architectural advantages over competing parallel supercomputers. For both production and grand challenge problems, sufficient disk and archival storage (ranging from $\mathrm{O}(100)$ GBytes to $\mathrm{O}(10)$ TBytes) is necessary to 
fully utilize the Meiko. Using the processor-local disk is key to having effective parallel distributed mesh generation and visualization tools.

- Network infrastructure in the way of Gigabit networks for distributed mesh generation and visualization as well as for archival storage will be required for production, general purpose supercomputing.

- In the near term, the parallel efficiency of FEM applications will be limited by immature software for both parallel $\mathrm{I} / \mathrm{O}$ and parallel solution of systems of linear equations. The current $1 / O$ options for unstructured grid applications pose sequential bottlenecks for parallel FEM codes.

- While there is work being done on equation solvers both inside and outside the laboratory, the specialized data structures required for FEM codes exceed the capabilities of methods developed for the solution of simple linear partial differential on a unit square.

In conclusion, the Meiko offers great potential for solving grand challenge problems, and has many architectural features which make it unique among parallel supercomputers. Not the least of these features are the processorlocal disk and the inter-processor network bandwidth of the machine. The laboratory should make every effort to exploit these features, but to do so will require an investment not only in terms of the machine, but in the laboratory network infrastructure and in the core computational competency areas of mesh generation, mesh partitioning, equation solving and visualization. With this infrastructure, the laboratory will be a world leader not only in parallel computing and networking, but also in its ability to solve grand challenge problems.

Acknowledgements The author wishes to thank Phillip M. Gresho, Gerald L. Goudreau, Richard Procassini, Carol Hoover, and James Maltby for their useful and constructive suggestions on this manuscript.

\section{References}

[1] R. G. Whirley, B.E. Englmann, "DYNA3D: A Nonlinear, Explicit, Three Dimensional Finite Element Code for Solid and Structural Mechanics - 
User Manual," Lawrence Livermore National Laboratory, UCRL-MA107254, Rev. 1, 1993.

[2] B. N. Maker, R. M. Ferencz, J. O. Hallquist, "NIKE3D, A Nonlinear, Implicit, Three-Dimensional Finite Element Code for Solid and Structural Mechanics - User's Manual," Lawrence Livermore National Laboratory, UCRL-MA-105268, 1991.

[3] M. Christon, A Vectorized 3-D Finite Element Model for Transient Simulation of Two-Phase Heat Transport with Phase Transformation and a Moving Interface, IEEE Supercomputing '90 , pp. 436-445, New York, New York, November 12-16, 1990.

[4] T. K. Agarwal, O. O. Storaasli, D. T. Nguyen, "A Parallel-Vector Algorithm for Rapid Structural Analysis on High-Performance Computers," AIAA 90-1149, April, 1990.

[5] The Federal High Performance Computing Program, Executive Office of the President, Office of Science and Technology, pp. 49-50, September 8, 1989.

[6] D. H. Bailey, "Twelve Ways to Fool the Masses when Giving Performance Results on Parallel Computers," Supercomputing Review, pp. 5455, August, 1991.

[7] R. W. Hockney, and C. R. Jesshope, Parallel Computers, Adam Hilger Ltd, pp. 53-56, 1981.

[8] Meiko, "Computing Surface: Vector Processing Element Overview," Meiko Scientific, 84-CB041, pp. 2-6, 1993.

[9] C. G. Hoover, J. D. Maltby, A. J. Degroot, R. G. Whirley, "ParaDyn: New Generation Solid/Structural Mechanics Codes for Massively Parallel Processors," Engineering Research, Development and Technology FY93, Lawrence Livermore National Laboratory, UCRL 53868-93, Section 2, pp. 11-14, May, 1994.

[10] M. A. Christon, "PING: An Explicit Finite Element Code for Linear Structural Acoustics - User Manual," Lawrence Livermore National Laboratory, UCRL-MA-114536, Draft - May, 1993. 
[11] M. A. Christon, "HYDRA: A Finite Element Code for Computational Fluid Dynamics - User Manual," Lawrence Livermore National Laboratory, in preparation, June, 1994.

[12] O. M. Lubeck, M. L. Simmons, H. J. Wasserman, "The Performance Realities of Massively Parallel Processors: A Case Study," proc. IEEE Supercomputing '92, pp. 403-412, 1992.

[13] P. J. Burns, M. Christon, R. Schweitzer, O. M. Lubeck, H. J. Wasserman, M. L. Simmons, and D. V. Pryor, "Vectorization of Monte Carlo Particle Transport: An Architectural Study Using the LANL Benchmark 'GAMTEB'," Proc. Supercomputing '89, IEEE Computer Society, pp. 10-20, 1989.

[14] A. Pothen, H. D. Simon, and K. P. Liou, "Partitioning Sparse Matrices with Eigenvectors of Graphs," SIAM J. Matrix Anal. Appl., Vol. 11, No. 3, pp. 430452, 1990 .

[15] B. Hendrickson, R. Leland, "An Improved Spectral Graph Partitioning Algorithm for Mapping Parallel Computations," Sandia National Laboratory, SAND92-1460, 1992.

[16] C. Farhat, and M. Lesoinne, "Automatic Partitioning of Unstructured Meshes for the Parallel Solution of Problems in Computational Mechanics," International Journal for Numerical Methods in Engineering, Vol. 36, pp. 745-764, 1993.

[17] Thinking Machines Corporation, CMSSL Relase Notes, Thinking Machines Corporation, Version 2.2, June, 1991.

[18] Meiko, "Computing Surface 2: Technical Overview," Meiko Scientific, S1002-10Mm115.01A, p. 10, 1993.

[19] D. D. Hardin, J. C. Compton, D. J. Dovey, G. W. Laguna, S. T. Pennock, "Massively Parallel Mesh Generation for Physics Codes," Lawrence Livermore National Laboratory, LDRD Proposal \# 94-SR-016, May, 1994.

[20] J. J. Dongarra, I. S. Duff, D. C. Sorensen, H. A. ven der Vorst, Solving Linear Systems on Vector and Shared Memory Computers, Society for Industrial and Applied Mathematics, Philadelphia, Pennsylvania, 1991. 
[21] R. Barrett, M. Berry, T. F. Chan, J. Demmel, J. Donato, J. Dongarra, V. Eijkhout, R. Poso, C. Romine, H. van der Vorst, Templates for the Solution of Linear Systems: Building Blocks for Iterative Methods, Society for Industrial and Applied Mathematics, Philadelphia, Pennsylvania, 1994.

[22] J. Choi, J. J. Dongarra, D. W. Walker, R. C. Whaley, "Scalapack Reference Manual, Parallel Factorization Routines (LU, QR, and Cholesky), and Parallel Reduction Routines (HRD, BRD, and TRD)," Oak Ridge National Laboratory, ORNL/TM-12470, April, 1994.

[23] A. Globus, E. Raible, "13 Ways to Say Nothing with Scientific Visualization," NASA Ames Research Center, Report RNR-92-006, February, 1992.

[24] R. Haimes, "pV3: A Distributed System for Large-Scale Unsteady CFD Visualization," AIAA 94-0321, January, 1994.

[25] D. E. Nielsen, Jr., "General Purpose Parallel Supercomputing," UCRLID-108228, Lawrence Livermore National Laboratory, June, 1991.

[26] A. Globus, "A Software Model for Visualization of Time Dependent 3-D Computational Fluid Dynamics Results," NASA Ames Research Center, Report RNR-92-031, November, 1992. 


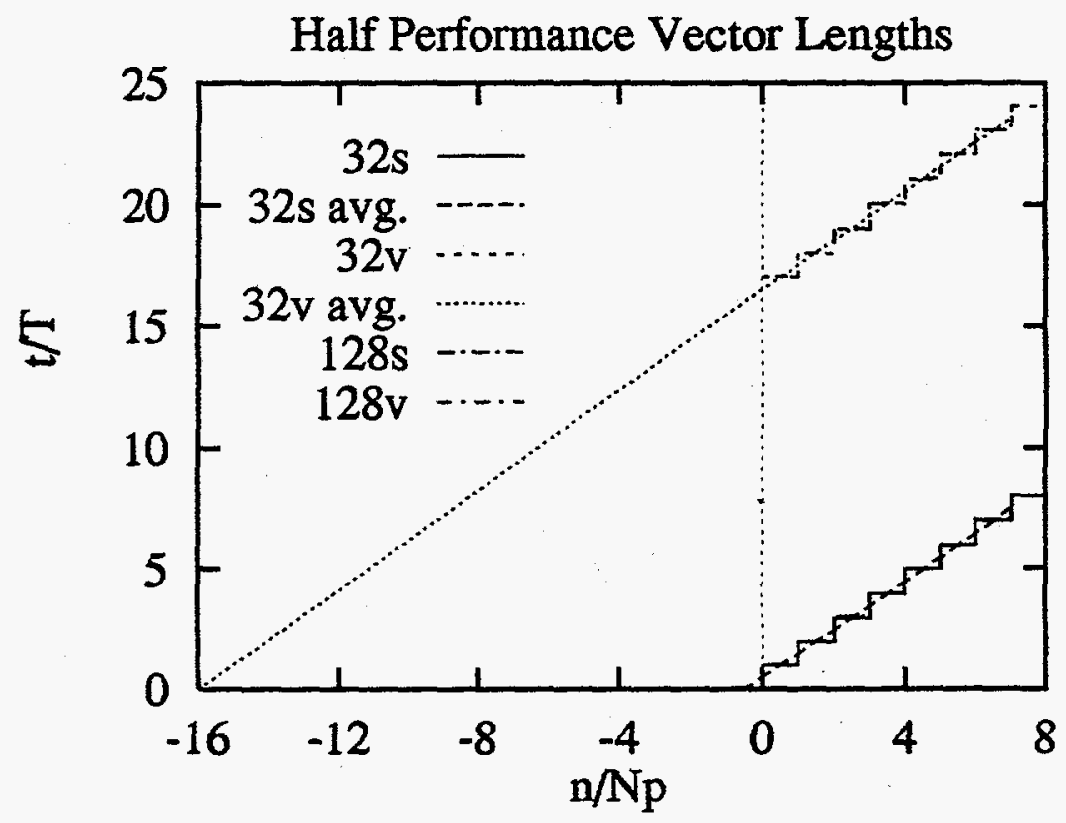

Figure 1: Network based distributed visualization model.

\begin{tabular}{||c|c|c||}
\hline \hline State Rate & \# of States & Storage [GBytes] \\
\hline \hline $10^{2}$ & $10^{3}$ & 80 \\
$10^{3}$ & $10^{2}$ & 8 \\
$10^{4}$ & 10 & 0.8 \\
\hline
\end{tabular}

Table 1: PING - State Disk Storage Requirements for a $10^{6}$ grid Point Problem. 